\title{
Immune oncology, immune responsiveness and the theory of everything
}

\author{
Tolga Turan', Deepti Kannan', Maulik Patel², J. Matthew Barnes', Sonia G. Tanlimco', Rongze Lu' ${ }^{1}$ Kyle Halliwill', \\ Sarah Kongpachith', Douglas E. Kline ${ }^{3}$, Wouter Hendrickx', Alessandra Cesano ${ }^{5}$, Lisa H. Butterfield ${ }^{6}$, \\ Howard L. Kaufman ${ }^{7}$, Thomas J. Hudson ${ }^{1}$, Davide Bedognetti ${ }^{4}$, Francesco Marincola ${ }^{1}$ and Josue Samayoa ${ }^{1 *}$ (i)
}

\begin{abstract}
Anti-cancer immunotherapy is encountering its own checkpoint. Responses are dramatic and long lasting but occur in a subset of tumors and are largely dependent upon the pre-existing immune contexture of individual cancers. Available data suggest that three landscapes best define the cancer microenvironment: immune-active, immunedeserted and immune-excluded. This trichotomy is observable across most solid tumors (although the frequency of each landscape varies depending on tumor tissue of origin) and is associated with cancer prognosis and response to checkpoint inhibitor therapy (CIT). Various gene signatures (e.g. Immunological Constant of Rejection - ICR and Tumor Inflammation Signature - TIS) that delineate these landscapes have been described by different groups. In an effort to explain the mechanisms of cancer immune responsiveness or resistance to CIT, several models have been proposed that are loosely associated with the three landscapes. Here, we propose a strategy to integrate compelling data from various paradigms into a "Theory of Everything". Founded upon this unified theory, we also propose the creation of a task force led by the Society for Immunotherapy of Cancer (SITC) aimed at systematically addressing salient questions relevant to cancer immune responsiveness and immune evasion. This multidisciplinary effort will encompass aspects of genetics, tumor cell biology, and immunology that are pertinent to the understanding of this multifaceted problem.
\end{abstract}

Keywords: Cancer immunotherapy, Checkpoint inhibitors, Immune resistance

\section{Premise and background}

Anti-cancer immunotherapy is encountering its own checkpoint. Responses are dramatic and long lasting but occur in a subset of tumors and are largely dependent upon the pre-existing immune contexture of individual cancers [1]. Current research is trying to determine why some cancers respond to CIT more than others and the reasons for individuals' variability within each indication $[2,3]$.

Several morphological observations based on immune histochemical analyses suggest that three immune landscapes best define distinct varieties of the cancer microenvironment: an immune-active, an opposite immune-deserted and an intermediate immune-excluded [4]. Across cancers, and among subtypes, the

\footnotetext{
* Correspondence: josue.samayoa@abbvie.com

Deepti Kannan and Francesco Marincola were employees of AbbVie at the time of the study but are no longer with the company.

'Immune-Oncology Discovery, AbbVie, Redwood City, CA, USA

Full list of author information is available at the end of the article
}

prevalence of each landscape may differ. Nevertheless, this trichotomy is observable across most solid tumors suggesting that convergent evolutionary adaptations determine the survival and growth of cancer in the immune competent host leading to predictable patterns determined by uniform immunological principles independent of the biology pertinent to distinct tumor tissue of origin. It is therefore reasonable to postulate that the mechanisms leading to cancer resistance to checkpoint blockade are similar across cancers deriving from different tissues. Functional characterization based on transcriptional analyses cannot distinguish structural differences. Thus a reductionist argument could be made that at the functional level cancers can simply be aggregated into immune-active or immune-silent clusters. Current work from our group suggests that most immune excluded cancer resemble functionally immune active tumors suggesting that the periphery immune cells interact with cancer cells (unpublished observation). 
We will refer to the mechanisms allowing persistence of cancer in the immune-active cluster as Compensatory Immune Resistance (CIRes) based on the assumption that lack of CIRes would prevent tumor survival against the host's immune response. Conversely, we refer to survival of cancer in the immune-deserted environment as Primary Immune Resistance (PIRes). In 2002, we proposed that human cancer immune responsiveness to antigen-specific vaccination administered in combination with systemic interleukin-2 is predetermined by a tumor microenvironment conducive to immune recognition [5] Likewise, recent observations suggest that CIT is most effective for the treatment of immune active tumors, where a tenuous balance between immuneeffector and immune-suppressive mechanisms determines outcomes [6-8].

To explain CIRes and PIRes, several phenomenologies have been described and models proposed that largely outnumber the fewer immune landscapes (Table 1). Such discrepancy can be explained in three ways: a) some models do not translate broadly across the majority of human cancers, b) there are subtler immune landscapes than those discernable by current approaches, or c) some models are redundant and describe different facets of the same pathophysiology. To solve this

Table 1 Principal models related to immune responsiveness

\begin{tabular}{lll}
\hline & Immune Landscape $^{\text {a }}$ & References \\
\hline WNT/BCatenin & Silent $(0.03)$ & {$[38,39]$} \\
MAPK Hypothesis & Silent $(0.001)$ & {$[10]$} \\
Immunogenic Cell Death & Active $(<0.001)$ & {$[19],[20,21]$} \\
Regulatory T cells & Active $(<0.001)$ & {$[24,25]$} \\
IL23-Th17 Axis & Active $(<0.001)$ & {$[26,41-44]$} \\
Myeloid Suppressor Cells & Active $(<0.001)$ & {$[50]$} \\
PI3K-y Signature & Active $(<0.01)$ & {$[52-55,63]$} \\
IDO/NOS Signature & Active $(<0.01)$ & {$[51,81,82]$} \\
SGK1 Signature & Ubiquitous & {$[56,57]$} \\
Shc1 signature & Ubiquitous & {$[62]$} \\
Barrier Molecules & Ubiquitous & {$[27,28]$} \\
Mesenchymal Transition & Ubiquitous & {$[29,30,83]$} \\
Cancer-Associated Fibroblasts & Ubiquitous & {$[31-35,84]$} \\
TAM receptor tyrosine kinases & Ubiquitous & {$[47,58-60,85]$} \\
Hypoxia/Adenosine suppression & Ubiquitous & {$[48,49]$} \\
TREX1clearence of Cytosolic DNA & NA & {$[86,87]$} \\
Checkpoint Cluster & Active $(<0.001)$ & {$[22,23]$} \\
oncogene addicted tumors & Silent & {$[11,68]$} \\
Epigenetic Regulation & Ubiquitous & {$[12,88-90]$} \\
\hline Distnt modes & &
\end{tabular}

${ }^{a}$ Distinct models have been assigned to either the Silent or the Active Landscape according to the results of the survey shown in Fig. 1. Ubiquitous refers to models that are not significantly associated with either immune landscape discrepancy, we surveyed human cancers through readily-available open-access information.

Marincola et al. [9] have previously described a transcriptional signature comprising the concordant activation of innate and adaptive immune effector mechanisms that is required for the occurrence of immune-mediated tissue-specific destruction. This represents a conserved mechanism determining destructive autoimmunity, clearance of pathogen-bearing cells during acute infection, acute allograft rejection, graftversus-host disease and rejection of cancer. Thus, the signature was termed: the Immunologic Constant of Rejection (ICR) [9]. The ICR signature was derived from bulk tumor transcriptome data sets, as they offer the most readily-available sample/data type and the easiest to apply in the clinic due to the ease of collection. The ICR signature was further trained to be representative of the broader signature as previously described [10] and is currently represented by twenty transcripts and four functional categories: CXCR3/CCR5 chemokines (including CXCL9, CXCL10, CCL5), Th1 signaling (including IFNG, IL12B, TBX21, CD8A, STAT1, IRF1, CD8B), effector (including GNLY, PRF1, GZMA, GZMB, $G Z M H$ ) and immune regulatory (including $C D 274$, CTLA4, FOXP3, IDO1, PDCD1) functions. The expression of these twenty representative genes is highly correlated with the extended ICR signature that includes approximately five-hundred transcripts and is representative of its main functional orientation as previously described [11, 12]. Importantly, the specific cell types in the tumor microenvironment expressing these genes will ultimately be relevant in elucidating the mechanistic link between the ICR and the immune responsiveness of cancer. It was subsequently observed that the ICR serves both as a positive predictor of responsiveness to immunotherapy and as a favorable prognostic marker for various tumor types $[6,10,13,14]$. This observation suggests that these related phenomena represent facets of a spectrum within the continuum of anti-cancer immune surveillance. Such continuity leads to the fair, though unproven, assumption that signatures predictive of prolonged survival may mark an immune-favorable cancer phenotype and serve as surrogate predictors of responsiveness to anti-cancer immunotherapies $[10,15]$. This assumption is also corroborated by recent reports suggesting that similar gene expression patterns predict response to CIT [6-8]. Specifically, Ayers et al. [6] using RNA from pre-treatment tumor samples of pembrolizumab-treated patients and the nCounter platform identified and validated a pan-tumor T-cell-inflamed gene signature correlating with clinical benefit. This tumor inflammation signature (TIS) contains IFN- $\gamma$-responsive genes (CD27, STAT1, IDO1, HLA-E, NKG7) related to antigen presentation (HLA-DQA1, 
HLA-DRB1, PSMB10, CMKLR1) chemokine expression (CCL5, CXCL9, CXCR6), cytotoxic activity (CD8A), and adaptive immune resistance (TIGIT, LAG3, CD274, $C D 276, P D C D 1 L G 2)$ and as such, is highly correlated to the ICR signature: composite scores for each signature calculated with ssGSEA software and compared according the expression values in the 999 breast cancer samples from TCGA were highly correlated $(r=0.98)$. The TIS has been developed into a clinical grade assay running on the nCounter platform currently being evaluated in ongoing pembrolizumab trials (3). We, therefore, developed a strategy to build a navigational map of cancer immunity with the primary purpose of assigning distinct immune responsive and resistant models to their respective immune-landscapes using the expression of twenty transcripts that are representative of the extended ICR signature.

Using the ICR signature [10], we queried the prognostic accuracy of a transcriptional data set of breast cancers from The Cancer Genome Atlas (TCGA) as a discovery platform and validated the findings on a second transcriptional set of breast cancers from the Gene Expression Omnibus (GEO) repository at the National Center for Biotechnology Information. The TCGA set encompasses RNA-seq-based transcriptional characterization of 999 breast cancer cases while the compilation of 10 GEO studies included 1728 cases of breast cancer (compiled in [16]) that were transcriptionally characterized utilizing a uniform Affymetrix platform. Both datasets were classified according to the coordinated expression of ICR transcripts [10].

ICR groups were ranked 1-4, according to the level of expression of the 20 representative ICR genes (Fig. 1). At the transcriptional level a dichotomy between Immune-active (ICR3-4) and immune-silent (ICR1-2) clusters was apparent [10]. Kaplan-Meier applied to the four ICR classes confirmed that ICR gene expression correlates with survival in breast cancer [10].

Subsequently, we collected transcriptional Signatures of Responsiveness (or Resistance) (sRes) as reported by other investigators (Table 1) and assessed them for their distribution within the four ICR groups (Fig. 1). The signatures tested and respective publication from which the transcript biomarkers were derived are available in Table 1. We recognize that the current collection of sRes is far from being comprehensive nor reflective of all proposed models of immune resistance and/or responsiveness. While further work is being entertained to refine and update the collection according to novel understanding of cancer immune biology, for the purpose of this commentary the current version sufficiently highlights the process that we are proposing.

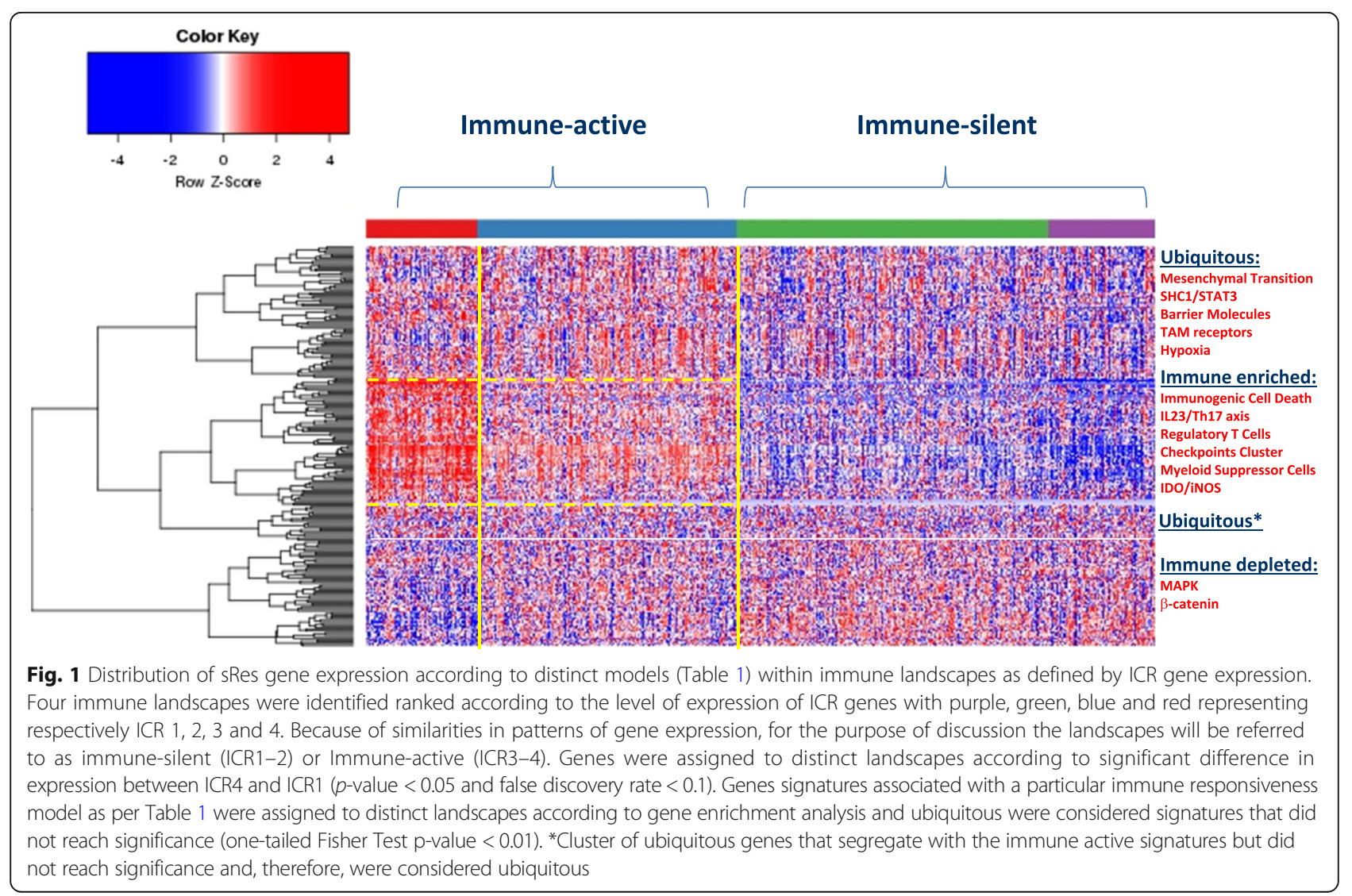


Self-organizing clustering of sRes signatures demonstrated a preferential distribution of immune suppressor activities such as those related to Th17-IL23 axis, T regulatory cells, checkpoint cluster, myeloid suppressor cells, IDO within the ICR4 and, to a lesser degree, the ICR3 immune landscapes (Fig. 1). This finding defines an immune phenotype of breast cancer enriched in concert with immune effector and immune suppressive mechanisms. Not surprisingly, the transcriptional signature representative of immunogenic cell death was included in the immune active landscape. This information presents a strong argument for the existence of CIRes mechanisms balancing immune pressure in these cancers' evolutionary processes.

Conversely, the immune depleted landscapes (ICR1 and ICR2) belonging to the immune silent cluster were best explained by PIRes, lacking evidence for the priming of a genuine immune response. The sRes of this cluster is enriched with transcripts in the PI3K $\gamma / \mathrm{SFK} /$ pGSK3/ $\beta$-catenin axis, and activation of the signal transducer and activator of transcription (STAT3). Coincidentally, these sRes are also associated with suppressive myeloid cell differentiation and activation of the IL-23/ Th17 axis. However, activation of the PI3K $\gamma / \mathrm{SFK} /$ pGSK3/ $\beta$-catenin axis does not correspond to activation of immunologic transcripts within the same cluster.

In conclusion, this survey suggested that:

1. In immune active tumors, signatures of immune suppression and activation are both present and this balance is responsible for CIRes in the ICR4, and to a lesser degree the ICR3, subclasses of breast cancer.

2. Immune active tumors (ICR3-4) are enriched in sRes and immunogenic signatures enriched for:
a. Immunogenic Cell Death activation
b. IL23/Th17,
c. Checkpoints cluster
d. Myeloid suppressor cells
e. Regulatory T cells
f. IDO

3. Immune-silent tumors are enriched with signatures reflecting activation of STAT3 and the PI3K $\gamma / \mathrm{SFK} /$ pGSK3/ $\beta$-catenin axis and their depletion of immune regulatory mechanisms argues for PIRes:
a. $\beta$-catenin
b. MAPK activation

Thus, the various models of immune resistance (Table 1) converge either into PIRes or CIRes. Interestingly, the CIRes signatures are co-expressed with those reflecting STING activation [17, 18] and immunogenic cell death [19-21]. This observation suggests that immunogenicity must be balanced by immune suppression in immune active tumors.
In an effort to move these in silico observations toward clinical validation and novel biology-based strategies of immune-modulation, new molecular tools which can be reproducibly applied in the clinic are needed. A possible candidate is the PanCancer IO 360 Gene Expression Panel (Nanostring), which allows for multiplexed targeted exploration of genes involved in the tumor-immune microenvironment, allowing for a multifaceted characterization of disease biology and interrogation of mechanisms of immune evasion. This panel was developed specifically for translational research and incorporates many of the PIRs and CIRes signatures including the ICR and the TIS.

\section{Discussion}

Several models have been proposed to explain proclivity or resistance of cancer in response to immunotherapy (Table 1). Effector T cell exhaustion is broadly observed in the tumor microenvironment manifesting through the expression of a cluster of immune checkpoints often concomitantly expressed in response to chronic interferon stimulation [22, 23]. In addition, it is well established that regulatory $\mathrm{T}$ cells balance immune effector mechanisms [24-26]. Other models propose blockade of immune cell homing to cancer tissue by barrier molecules, chemo-inhibitory mechanisms, and by epigenetic silencing of chemokines (CCL5, CXCL9, and CXCL1O), Th1 signaling molecules and antigen processing machinery components [12, 27-37].

Other immune resistance models point to alterations of cancer cell signaling that result in secondary dysregulation of myeloid cell function. Cancer-intrinsic $\beta$-catenin signaling defects disrupt chemo-attraction of dendritic cells (DCs) and, consequently, antigen presentation in the context of immunogenic cell death [21, 38-40]. In addition, polarization of DCs toward a tolerogenic, IL23 producing phenotype leading to Th17 polarization was described in experimental animal models and in human samples [26, 41-46]. Suppression of anti-cancer immunity has also been attributed to the TAM receptor tyrosine kinase family members that mediate efferocytosis and negative regulation of DC activity [47]. Similarly, hypoxia can drive immune suppression by inducing tolerogenic myeloid DC polarization [48, 49]. Finally, myeloid cell biology is responsible for the immune regulation of the cancer microenvironment through the upregulation of metabolizing enzymes such as arginase and indoleamine 2,3-dioxygenase, which can negatively impact $\mathrm{T}$ cell function $[50,51]$.

The phenotype of suppressive myeloid cells in the microenvironment is often attributed to activation of the PI3K $\gamma /$ SFK/pGSK3/ $\beta$-catenin axis (Fig. 2). Phosphoinositide3kinase-gamma (PI3K $\gamma)$ can act as a molecular switch that triggers immune suppressive mechanisms in myeloid DCs 


\section{The "Two-Option Choice" (TOC) determinism in the natural history of cancer: A conserved evolutionary crossroad for cancer survival}

Immune silent

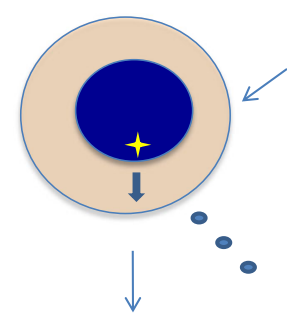

Apoptotic recycling Epigenetic silencing Oncogene-driven addiction

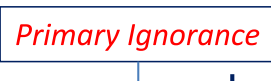

Low Mutational Burden High Limited Transcriptional Activity Broad Low Neo-epitope Frequency High High Stromal Composition Low

\section{Tumor Growth}

Fig. 2 Dichotomy in the Myeloid-Centric Hypothesis of immune resistance: the same pathway is relevant to myeloid cell differentiation as well as intrinsic oncogenic activation (in red boxes are included models included in Table 1). It is currently unclear how the two interpretations diverge vs relate to each other and further characterization of the single cell level will need to be entertained to clarify this point

$[52,53]$. At the same time, alteration of PI3K functional components plays a widespread role in tumorigenesis [54]. Downstream phosphorylation of serum and glucocorticoid kinase 1 (SGK1) by the PI3K/PDK1 cascade leads to activation of glycogen synthase kinase 3 beta (GSK3 $\beta$ ) and subsequently $\beta$-catenin [55-57]. Interestingly, most studies describing dysregulation of the PI3K $\gamma /$ SFK/pGSK3/ $\beta$-catenin axis refer to abnormalities intrinsic to tumor cells, although the same pathway can play an important role in myeloid suppressor DC induction and immune suppression downstream of the TAM receptor tyrosine kinases [58-60]. Converging on the same pathway, hypoxia inducible factors (HIF1 $\alpha)$ signal through the SGK $3 \beta / \beta$-catenin axis promoting cancer cell stemness and immune suppression [48, 49, 61] (Fig. 2).

An upstream inducer of PI3K $\gamma$ stimulation is the scaffold protein $\mathrm{SHC} 1$ that shifts the balance between STAT1 and STAT3 activation in favor of the latter, promoting immune suppression [62]. The context in which activation of SHC1 preferentially regulates myeloid DC polarization versus cancer cell signaling remains unclear. Similarly, loss of protein tyrosine phosphatase non-receptor type 2 (PTPN2) function that inhibits PI3K $\gamma$ signaling is associated with activation of the tumorigenic pathway, while at the same time can modulate $\mathrm{T}$ cell function through $\mathrm{mDC}$ activation $[63,64]$ and induction of Th17 polarization [65, 66]. Finally activation of the mitogen-activated protein kinases (MAPKs) programs is consistently observed in immune silent tumors and is associated with a respective mutational signature [10].
Therefore, it may be that most models of immune resistance are based on a diverse interpretation of the disruption of the PI3K $\gamma / \mathrm{SFK} / \mathrm{pGSK} 3 / \beta$-catenin pathway: one centered on tumorigenesis and the other on myeloid cell biology however it is currently unclear whether the two mechanisms are mutually exclusive or can be observed in association in the immune active tumors. This question can only be solved by morphological documentation of cell-specific activation of the pathway either by immunohistochemistry or by single cell transcriptional analysis. However, according to our results and the published literature [10, 38, 67], it appears that the former interpretation pertains most prominently to the immune silent cluster (PIRes) while the latter appears to be most likely pertaining to the immune active (CIRes, Fig. 2).

These results may bear remarkable impact for the design of combination therapies. It appears that, at least in breast cancer, therapeutic combinations directed against immune regulatory mechanism (i.e. checkpoint blockade, IL-23/ Th17, TAM receptor kinases, hypoxia factors or IDO inhibitors) will modulate and possibly enhance responsiveness of cancers with CIRes (immune active cluster) but will be unlikely to work in the context of immune silent cancers of the PIRes phenotype unless complimentary efforts are made to disrupt the non-immunogenic landscape to convert it into an immunogenic one.

We hypothesize that immune silent tumors evolve by employing a strictly essential interface of interactions with the host's stroma that limits immune cell recognition. 
This may be due to the selection of a growth process devoid of immunogenic cell death (Fig. 1). Thus, these "clean" tumors evolve through the selection of cancer cells that adopt refined growth mechanisms reduced to the bare necessities of life. Indeed, preclinical and clinical data focused on molecular subtypes of clinically-validated oncogene-addicted tumors (e.g., ALK+, EGFR+, BRAFV600E + , NTRK-rearranged tumors) indicate that these tumors often portray minimal CD8+ T cell infiltration along with reduced expression of immunosuppressive factors [11, 68]. These molecular subtypes of EGFRmutated or ALK+ non-small cell lung cancer (NSCLC) serve as a perfect clinically validated example of "clean tumors" as these tumors usually do not have high mutational burden, occur in younger patients, and in nonsmokers. This is supported by recent evidence which demonstrates that presence of oncogenic driver mutations in NSCLC, such as EGFR, ALK, ROS1, RET fusions and C-MET exon 14 skipping is associated with lower mutational burden (Mohamed E. Salem, ASCO presentation 2017, http://abstracts.asco.org/199/AbstView_199_ 184601.html). This hypothesis is further corroborated by the observation that these tumors bear a low prevalence of mutations in oncogenes suggesting a more orderly growth process [10]. It is, therefore, reasonable to suppose that the growth of clean ("oncogene addicted") tumors is dependent on activation of specific pathways (e.g. the PI3K $\gamma / \mathrm{SFK} / \mathrm{pGSK} 3 / \beta$-catenin axis) that avoid immune recognition. Thus, we propose that the natural history of cancer is shaped at the crossroad of two biologies by a "Two-Option Choice": 1) immunogenic tumors evolve through a disorderly trial-and-error accumulation of oncogenic processes generated by their intrinsic genetic instability that leads to a broader number of host-immune interactions. These tumors can, therefore, only survive in the immune competent host when immune suppressive mechanisms balance the immune reaction, 2) silent tumors follow a more orderly process with a sequential accumulation of essential genetic traits and can grow undisturbed by the immune system (Fig. 3). Since the latter appear to depend on a leaner carcinogenesis, it may be reasonable to postulate that disruption of this delicate survival skill may induce messier cancer biology prone to immunogenic cell death. Whether this is true remains to be tested. Turning an immune silent into immune active tumor microenvironment, even temporarily, may serve a critical therapeutic role opening the door for immunotherapy strategies. This in turn may be critical because successful anti-cancer immunotherapy induces durable tumor regression and immune memory more frequently.

In conclusion, we propose a systematic, hypothesisdriven task force led by SITC to prioritize and address the salient questions related to cancer immune responsiveness based on a deeper understanding of the cancer cell biology that orchestrates distinct immune landscapes. The task force should address outstanding questions to identify conserved versus peculiar patterns

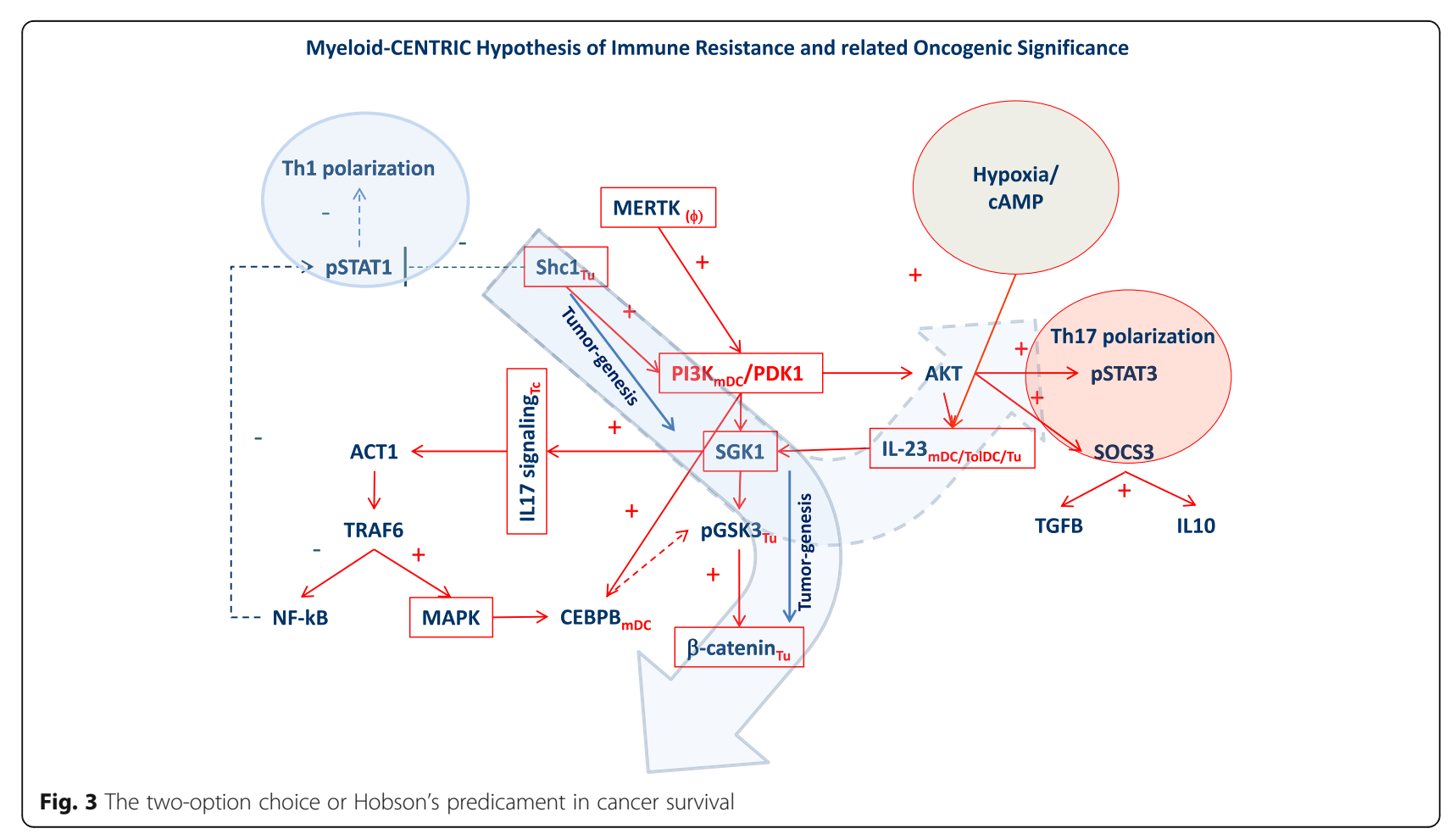


of immune interaction between the host and cancers of different ontology. The role that the genetic background of the host or micro environmental modifiers play in regulating cancer-immune biology should be addressed following appropriate stepwise approaches [69]. In addition, a deeper understanding of the evolutionary processes shaping the development of cancer in the immune competent host may offer a simplified understanding of conserved mechanisms of cancer survival and consequently help the identification of a broad range of therapeutics that can target dominant pathways leading to immunogenic cancer cell death. A clearer qualification of the role played by adaptive versus innate mechanisms in initiating immune activation should be considered. Two non-exclusive yet divergent lines of thought are raised to explain immunogenic cancer biology: on one side the high prevalence of neo-epitopes predicted by the higher mutational burden observed in immunogenic tumors positions adaptive immune recognition at the forefront of immune activation [70-74]. Conversely, immunogenic cell death may primarily drive inflammation with secondary recruitment of immune cells $[20,21,75,76]$. The role that each mechanism plays in human cancer biology, and its implication for therapeutic intervention, remains to be clarified, and better integrated tools may improve our holistic understanding of the underlying cancer-immune biology thus facilitating novel biology-based combinational therapeutic strategies.

Finally, better in vivo (genetically engineered and/or syngeneic) rodent models for the screening of therapeutic strategies should be better characterized [77-79]. Some animal models may be reflective of immuneactivated landscapes and be most relevant for the definition of therapies combining immune modulatory agents. Other animal models may more closely resemble the biology of immune-silent cancers and would be best utilized to identify therapies that can initiate an immune response before immunomodulatory agents are introduced sequentially and/or combinatorically. The availability of complimentary mouse/human paired panels would largely facilitate such efforts. To our knowledge, little has been done so far to match mouse models to corresponding human immuno-oncology phenotypes following the perspective proposed by this unified theory of everything.

The Taskforce will define its goals and future activities in the occasion of a foundational workshop to be held in San Francisco on May 14-15 2018 (SITC Cancer Immune Responsiveness Workshop).

The topics to be discussed will include:

- Interactions between tumor evolution in the immune competent host and the resulting immune landscape
- Identification of common pathways that could be interrogated and targeted to better understand and increase immunogenicity among silent or 'cold' cancers

- Mechanistic understanding of parameters that could predict immune response to different cancer immunotherapies

- Development of animal models that accurately reflect the immune landscape in 'hot' versus 'cold' human tumors

This workshop will be held in tandem with the SITC Biomarkers Workshop to be held subsequently on May 16-17 in the same premise as part of a strong interest by SITC and other organizations [80] to deepen the understanding of cancer immune biology particularly in association with clinical trial development: (SITC Biomarkers Workshop).

\section{Methods}

All data download, processing and analyses were done in $\mathrm{R}$ programming environment and as described in Hendrickx et al. [11]. For the unsupervised clustering of the TOE genes (Additional file 1), modified distance and hierarchical clustering functions were used. Specifically the distance between 2 genes was defined as 1-"Correlation Coefficient (Spearman)" and for the hierarchical clustering function "Ward.D2" method was used.

Composite correlation between the ICR and the TIS signature was assessed by calculating a cumulative score for each gene included in the respective signature using ssGSEA method form GSVA package and correlating the scores in the breast cancer TCGA data set according to Spearman Correlation.

The metrics used when assigning genes to silent, active and ubiquitous groups are derived from differential expression statistics between ICR1 and ICR4 samples. The genes are assigned to the active cluster if they have significantly higher expression levels in ICR4 samples ( $p$-value $<0.05$ and FDR $<0.1$ ). Similarly the genes are assigned to the silent cluster if they have significantly higher expression levels in ICR1 samples ( $p$-value $<0.05$ and FDR $<0$. $1)$. If the genes do not pass these cutoffs they are grouped as "Ubiquitous". Geneset enrichment for each signature belonging to individual models of immune resistance (Table 1) against ICR1 and ICR4 clusters was assessed using one-tailed Fisher's exact test.

In the analyses and corresponding heatmaps, the genes that were identified in multiple signatures were plotted as one, so each gene in the heatmap is unique. When the ICR direction is inferred for each signature, the repeated genes contributed to each signature with the same statistics. 


\section{Additional file}

Additional file 1: List of individual genes and corresponding models related to immune suppression used for the Theory of Everything (TOE). (TXT $11 \mathrm{~kb}$ )

\section{Abbreviations}

CIRes: Compensatory Immune Resistance; DC: Dendritic Cell; GEM: Genetically Modified Mouse Models; GEO: Gene Expression Omnibus; GSK: Glycogen synthase kinase; ICR: Immunologic Constant of Rejection; IDO: Indoleamine 2,3-dioxygenase; MAPK: Mitogen-activated protein kinase; PI3K: Phosphoinositide3-kinase-gamma; PIRes: Primary Immune Resistance; PTPN2: Protein tyrosine phosphatase non-receptor type 2; SGK: Serum and glucocorticoid kinase; sRes: Signatures of Resistance; STAT: Signal transducer and activator of transcription 1; TCGA: The Cancer Genome Atlas; TCIA: The Cancer Immunome Atlas; TOC: Two-Option Choice; TOE: Theory of Everything

\section{Funding}

The design, study conduct, and financial support for this research were provided by AbbVie. AbbVie participated in the interpretation of data, review, and approval of the publication.

\section{Availability of data and materials}

The datasets used and/or analysed during the current study are available from the corresponding author on reasonable request.

\begin{abstract}
AbbVie disclosures
Tolga Turan, Maulik Patel, Mathew Barnes, Sonia Tanlimco, Rongze Lu, Sarah Kongpachith, Kyle Halliwill, Douglas Kline, Thomas Hudson, and Josue Samayoa are all employees of AbbVie. Deepti Kannan and Francesco Marincola were employees of AbbVie at the time of the study. Dr. Howard Kaufman, Dr. Lisa Butterfield, Dr. Alessandra Cesano, Dr. Davide Bedognetti, and Dr. Wouter Hendrickx have no current or prior affiliation with AbbVie beyond the current study. The design, study conduct, and financial support for this research were provided by AbbVie. AbbVie participated in the interpretation of data, review, and approval of the publication.
\end{abstract}

\section{Authors' contributions}

Tolga Turan and Deepti Kannan were primarily responsible for the processing and analysis of all the data used in this study. Franco Marincola was a major contributor in writing the manuscript. All authors contributed equally to the conception and design, analysis and interpretation of data and have been involved in revising it critically for important intellectual content. All authors read and approved the final manuscript.

\section{Ethics approval and consent to participate}

The results shown here are based upon data generated by the TCGA Research Network: http://cancergenome.nih.gov/. All of the data used for this study was obtained as described in Hendrickx et al., 2017.

\section{Competing interests}

Dr. Howard L. Kaufman has served on advisory boards for Amgen, Celldex, Compass Therapeutics, EMD Serono, Merck, Prometheus and Turnstone Biologics. He serves on a speaker's bureau for Merck but all honoraria are returned to Rutgers University. Alessandra Cesano is Chief Medical Officer at Nanostring whose platform is mentioned in the text.

\section{Publisher's Note}

Springer Nature remains neutral with regard to jurisdictional claims in published maps and institutional affiliations.

\footnotetext{
Author details

${ }^{1}$ Immune-Oncology Discovery, AbbVie, Redwood City, CA, USA. ${ }^{2}$ Clinical Pharmacology and Pharmacometrics, Abbvie, Redwood City, CA, USA ${ }^{3}$ Immune-Oncology, AbbVie, North Chicago, IL, USA. ${ }^{4}$ Tumor Biology, Immunology, and Therapy Section, Division of Translational Medicine, Research Branch, Sidra Medical and Research Center, Doha, Qatar. ${ }^{5}$ Nanostring Technologies, Inc., Seattle, WA, USA. ${ }^{6}$ UPMC Hillman Cancer Center, University of Pittsburgh, Pittsburgh, PA, USA. ${ }^{7}$ Departments of Surgery and Medicine, Rutgers University, New Jersey, NJ, USA.
}

Received: 22 November 2017 Accepted: 11 May 2018

Published online: 05 June 2018

\section{References}

1. Gong J, et al. Development of PD-1 and PD-L1 inhibitors as a form of cancer immunotherapy: a comprehensive review of registration trials and future considerations. J Immunother Cancer. 2018;6(1):8.

2. Spranger $\mathrm{S}$, et al. Tumor and host factors controlling antitumor immunity and efficacy of Cancer immunotherapy. Adv Immunol. 2016;130:75-93.

3. Emens LA, et al. Cancer immunotherapy: opportunities and challenges in the rapidly evolving clinical landscape. Eur J Cancer. 2017:81:116-29.

4. Chen DS, Mellman I. Elements of cancer immunity and the cancer-immune set point. Nature. 2017;541(7637):321-30.

5. Wang $E$, et al. Prospective molecular profiling of melanoma metastases suggests classifiers of immune responsiveness. Cancer Res. 2002;62(13): 3581-6.

6. Ayers $\mathrm{M}$, et al. IFN-gamma-related mRNA profile predicts clinical response to PD-1 blockade. J Clin Invest. 2017;127(8):2930-40

7. Prat $\mathrm{A}$, et al. Immune-related gene expression profiling after PD-1 blockade in non-small cell lung carcinoma, head and neck squamous cell carcinoma, and melanoma. Cancer Res. 2017;77(13):3540-50.

8. Charoentong $\mathrm{P}$, et al. Pan-cancer Immunogenomic analyses reveal genotype-Immunophenotype relationships and predictors of response to checkpoint blockade. Cell Rep. 2017:18(1):248-62.

9. Wang E, Worschech A, Marincola FM. The immunologic constant of rejection. Trends Immunol. 2008;29(6):256-62.

10. Hendrickx $W$, et al. Identification of genetic determinants of breast cancer immune phenotypes by integrative genome-scale analysis. Oncoimmunology. 2017;6(2):e1253654.

11. Akbay EA, et al. Activation of the PD-1 pathway contributes to immune escape in EGFR-driven lung tumors. Cancer Discov. 2013;3(12):1355-63.

12. Guerriero JL, et al. Class Ila HDAC inhibition reduces breast tumours and metastases through anti-tumour macrophages. Nature. 2017;543(7645): 428-32.

13. Galon J, et al. The continuum of cancer immunosurveillance: prognostic predictive, and mechanistic signatures. Immunity. 2013;39(1):11-26.

14. Bedognetti $D$, et al. Prognostic and predictive immune gene signatures in breast cancer. Curr Opin Oncol. 2015;27(6):433-44.

15. Miller $L D$, et al. Immunogenic subtypes of breast Cancer delineated by gene classifiers of immune responsiveness. Cancer Immunol Res. 2016;4(7):600-10.

16. Nagalla $\mathrm{S}$, et al. Interactions between immunity, proliferation and molecular subtype in breast cancer prognosis. Genome Biol. 2013;14(4):R34.

17. Barber GN. STING: infection, inflammation and cancer. Nat Rev Immunol. 2015:15(12):760-70.

18. Corrales $L$, et al. The host STING pathway at the interface of cancer and immunity. J Clin Invest. 2016;126(7):2404-11.

19. Galluzzi $L$, et al. Immunogenic cell death in cancer and infectious disease. Nat Rev Immunol. 2017:17(2):97-111.

20. Wegner KW, Saleh D, Degterev A. Complex pathologic roles of RIPK1 and RIPK3: moving beyond Necroptosis. Trends Pharmacol Sci. 2017;38(3):202-25.

21. Matt S, Hofmann TG. The DNA damage-induced cell death response: a roadmap to kill cancer cells. Cell Mol Life Sci. 2016;73(15):2829-50.

22. Benci JL, et al. Tumor Interferon Signaling Regulates a Multigenic Resistance Program to Immune Checkpoint Blockade. Cell. 2016;167(6):1540-1554.e12.

23. Koyama S, et al. Adaptive resistance to therapeutic PD-1 blockade is associated with upregulation of alternative immune checkpoints. Nat Commun. 2016:7:10501.

24. Abd Al Samid M, et al. Combining FoxP3 and Helios with GARP/LAP markers can identify expanded Treg subsets in cancer patients. Oncotarget. 2016;7(12):14083-94

25. Elkord E, Abd Al Samid M, Chaudhary B. Helios, and not FoxP3, is the marker of activated Tregs expressing GARP/LAP. Oncotarget. 2015;6(24):20026-36.

26. Tosolini $M$, et al. Clinical impact of different classes of infiltrating $T$ cytotoxic and helper cells (Th1, th2, treg, th17) in patients with colorectal cancer. Cancer Res. 2011;71(4):1263-71

27. Salerno EP, et al. Human melanomas and ovarian cancers overexpressing mechanical barrier molecule genes lack immune signatures and have increased patient mortality risk. Oncoimmunology. 2016:5(12):e1240857.

28. Buckanovich RJ, et al. Endothelin B receptor mediates the endothelial barrier to T cell homing to tumors and disables immune therapy. Nat Med. 2008;14(1):28-36. 
29. Hugo W, et al. Genomic and Transcriptomic features of response to anti-PD1 therapy in metastatic melanoma. Cell. 2016;165(1):35-44.

30. Shields BD, et al. Indicators of responsiveness to immune checkpoint inhibitors. Sci Rep. 2017;7(1):807.

31. Feig C, et al. Targeting CXCL12 from FAP-expressing carcinoma-associated fibroblasts synergizes with anti-PD-L1 immunotherapy in pancreatic cancer. Proc Natl Acad Sci U S A. 2013;110(50):20212-7.

32. Kraman $M$, et al. Suppression of antitumor immunity by stromal cells expressing fibroblast activation protein-alpha. Science. 2010;330(6005):827-30.

33. Ohlund D, Elyada E, Tuveson D. Fibroblast heterogeneity in the cancer wound. J Exp Med. 2014;211(8):1503-23.

34. Ohlund D, et al. Distinct populations of inflammatory fibroblasts and myofibroblasts in pancreatic cancer. J Exp Med. 2017;214(3):579-96.

35. Ozdemir BC, et al. Depletion of carcinoma-associated fibroblasts and fibrosis induces immunosuppression and accelerates pancreas cancer with reduced survival. Cancer Cell. 2014;25(6):719-34.

36. Vo DD, et al. Enhanced antitumor activity induced by adoptive T-cell transfer and adjunctive use of the histone deacetylase inhibitor LAQ824. Cancer Res. 2009;69(22):8693-9.

37. Zheng $\mathrm{H}$, et al. HDAC inhibitors enhance T-cell chemokine expression and augment response to PD-1 immunotherapy in lung adenocarcinoma. Clin Cancer Res. 2016;22(16):4119-32.

38. Spranger S, Bao R, Gajewski TF. Melanoma-intrinsic beta-catenin signalling prevents anti-tumour immunity. Nature. 2015;523(7559):231-5.

39. Corrales $\mathrm{L}$, et al. Innate immune signaling and regulation in cancer immunotherapy. Cell Res. 2017;27(1):96-108.

40. Spranger S, Gajewski TF. A new paradigm for tumor immune escape: betacatenin-driven immune exclusion. J Immunother Cancer. 2015:3:43.

41. Coccia M, et al. IL-1beta mediates chronic intestinal inflammation by promoting the accumulation of IL-17A secreting innate lymphoid cells and CD4(+) Th17 cells. J Exp Med. 2012;209(9):1595-609.

42. Kirchberger $S$, et al. Innate lymphoid cells sustain colon cancer through production of interleukin-22 in a mouse model. J Exp Med. 2013;210(5): 917-31

43. Alinejad V, et al. The role of IL17B-IL17RB signaling pathway in breast cancer. Biomed Pharmacother. 2017:88:795-803.

44. Ngiow SF, Teng MW, Smyth MJ. A balance of interleukin-12 and -23 in cancer. Trends Immunol. 2013:34(11):548-55.

45. Spivey $\mathrm{TL}$, et al. The stable traits of melanoma genetics: an alternate approach to target discovery. BMC Genomics. 2012;13:156.

46. Dixon $\mathrm{KO}$, et al. Phagocytosis of apoptotic or necrotic cells differentially regulates the transcriptional expression of IL-12 family members in dendritic cells. J Leukoc Biol. 2014;96(2):313-24.

47. Akalu YT, Rothlin CV, Ghosh S. TAM receptor tyrosine kinases as emerging targets of innate immune checkpoint blockade for cancer therapy. Immunol Rev. 2017;276(1):165-77.

48. Hatfield SM, Sitkovsky M. A2A adenosine receptor antagonists to weaken the hypoxia-HIF-1alpha driven immunosuppression and improve immunotherapies of cancer. Curr Opin Pharmacol. 2016;29:90-6.

49. Hu CJ, et al. Differential roles of hypoxia-inducible factor 1alpha (HIF-1alpha) and HIF-2alpha in hypoxic gene regulation. Mol Cell Biol. 2003;23(24):9361-74.

50. Munn DH, Bronte V. Immune suppressive mechanisms in the tumor microenvironment. Curr Opin Immunol. 2016:39:1-6.

51. Mondanelli $\mathrm{G}$, et al. The immune regulation in cancer by the amino acid metabolizing enzymes ARG and IDO. Curr Opin Pharmacol. 2017:35:30-9.

52. De Henau O, et al. Overcoming resistance to checkpoint blockade therapy by targeting PI3Kgamma in myeloid cells. Nature. 2016;539(7629):443-7.

53. Kaneda MM, et al. PI3Kgamma is a molecular switch that controls immune suppression. Nature. 2016;539(7629):437-42.

54. Daragmeh J, et al. Analysis of PI3K pathway components in human cancers. Oncol Lett. 2016;11(4):2913-8.

55. Liu $P$, et al. Targeting the phosphoinositide 3-kinase pathway in cancer. Nat Rev Drug Discov. 2009;8(8):627-44.

56. Di Cristofano A. SGK1: the dark side of PI3K signaling. Curr Top Dev Biol. 2017;123:49-71

57. Xiaobo $Y$, et al. Serum and glucocorticoid kinase 1 promoted the growth and migration of non-small cell lung cancer cells. Gene. 2016;576(1 Pt 2): 339-46.

58. Crittenden MR, et al. Mertk on tumor macrophages is a therapeutic target to prevent tumor recurrence following radiation therapy. Oncotarget. 2016; 7(48):78653-66.
59. Zhang B, et al. Mer receptor tyrosine kinase negatively regulates lipoteichoic acid-induced inflammatory response via PI3K/Akt and SOCS3. Mol Immunol. 2016;76:98-107

60. Crittenden MR, et al. Expression of NF-kappaB p50 in tumor stroma limits the control of tumors by radiation therapy. PLoS One. 2012;7(6):e39295.

61. Vadde $\mathrm{R}$, et al. Role of hypoxia-inducible factors (HIF) in the maintenance of stemness and malignancy of colorectal cancer. Crit Rev Oncol Hematol. 2017;113:22-7.

62. Ahn R, et al. The Shc1 adaptor simultaneously balances Stat1 and Stat3 activity to promote breast cancer immune suppression. Nat Commun. 2017; 8:14638.

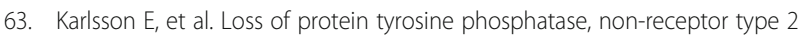
is associated with activation of AKT and tamoxifen resistance in breast cancer. Breast Cancer Res Treat. 2015;153(1):31-40.

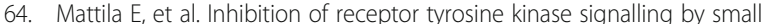
molecule agonist of T-cell protein tyrosine phosphatase. BMC Cancer. 2010;10:7

65. Wu R, et al. MicroRNA-448 promotes multiple sclerosis development through induction of Th17 response through targeting protein tyrosine phosphatase non-receptor type 2 (PTPN2). Biochem Biophys Res Commun. 2017:486(3):759-66.

66. Spalinger MR, et al. PTPN2 controls differentiation of CD4(+) T cells and limits intestinal inflammation and intestinal dysbiosis. Mucosal Immunol. 2015;8(4):918-29.

67. Spranger S, Gajewski TF. Tumor-intrinsic oncogene pathways mediating immune avoidance. Oncoimmunology. 2016;5(3):e1086862.

68. Gainor JF, et al. EGFR mutations and ALK rearrangements are associated with low response rates to PD-1 pathway blockade in non-small cell lung Cancer: a retrospective analysis. Clin Cancer Res. 2016;22(18):4585-93.

69. Wang E, Uccellini L, Marincola FM. A genetic inference on cancer immune responsiveness. Oncoimmunology. 2012;1(4):520-5.

70. Brennick CA, et al. Neoepitopes as cancer immunotherapy targets: key challenges and opportunities. Immunotherapy. 2017;9(4):361-71.

71. Tureci $\mathrm{O}$, et al. Targeting the heterogeneity of Cancer with individualized Neoepitope vaccines. Clin Cancer Res. 2016;22(8):1885-96.

72. Gubin MM, et al. Checkpoint blockade cancer immunotherapy targets tumour-specific mutant antigens. Nature. 2014;515(7528):577-81.

73. Ward JP, Gubin MM, Schreiber RD. The role of Neoantigens in naturally occurring and therapeutically induced immune responses to Cancer. Adv Immunol. 2016;130:25-74.

74. Noguchi T, et al. Temporally distinct PD-L1 expression by tumor and host cells contributes to immune escape. Cancer Immunol Res. 2017;5(2):106-17.

75. Galluzzi L, et al. Activating autophagy to potentiate immunogenic chemotherapy and radiation therapy. Nat Rev Clin Oncol. 2017;14(4):247-58.

76. Galluzzi L, et al. Regulated cell death and adaptive stress responses. Cell Mo Life Sci. 2016;73(11-12):2405-10.

77. Mosely SI, et al. Rational selection of syngeneic preclinical tumor models for immunotherapeutic drug discovery. Cancer Immunol Res. 2017;5(1):29-41.

78. Decker WK, et al. Cancer immunotherapy: historical perspective of a clinical revolution and emerging preclinical animal models. Front Immunol. 2017:8:829.

79. Maykel J, et al. NOD-scidll2rg (tm1Wjl) and NOD-Rag1 (null) $\| 2$ rg (tm1Wjl) : a model for stromal cell-tumor cell interaction for human colon cancer. Dig Dis Sci. 2014;59(6):1169-79.

80. Masucci GV, et al. The need for a network to establish and validate predictive biomarkers in cancer immunotherapy. J Transl Med. 2017;15(1):223.

81. Munn DH, Mellor AL. IDO in the tumor microenvironment: inflammation, counter-regulation, and tolerance. Trends Immunol. 2016;37(3):193-207.

82. Liu Q, et al. Melanoma NOS1 expression promotes dysfunctional IFN signaling. J Clin Invest. 2014:124(5):2147-59.

83. Herbst RS, et al. Predictive correlates of response to the anti-PD-L1 antibody MPDL3280A in cancer patients. Nature. 2014:515(7528):563-7.

84. Saleh SMI, et al. Identification of interacting stromal axes in triple-negative breast Cancer. Cancer Res. 2017:77(17):4673-83.

85. Grabiec AM, Hussell T. The role of airway macrophages in apoptotic cel clearance following acute and chronic lung inflammation. Semin Immunopathol. 2016;38(4):409-23.

86. Demaria S, Golden EB, Formenti SC. Role of local radiation therapy in Cancer immunotherapy. JAMA Oncol. 2015;1(9):1325-32.

87. Vanpouille-Box $\mathrm{C}$, et al. DNA exonuclease Trex 1 regulates radiotherapyinduced tumour immunogenicity. Nat Commun. 2017:8:15618. 
88. Place AE, Jin Huh S, Polyak K. The microenvironment in breast cancer progression: biology and implications for treatment. Breast Cancer Res 2011;13(6):227.

89. Adeegbe DO, et al. Synergistic Immunostimulatory effects and therapeutic benefit of combined histone Deacetylase and Bromodomain inhibition in non-small cell lung Cancer. Cancer Discov. 2017;7(8):852-67.

90. Mondino A, Vella G, Icardi L. Targeting the tumor and its associated stroma: one and one can make three in adoptive T cell therapy of solid tumors. Cytokine Growth Factor Rev. 2017;36:57-65

- fast, convenient online submission

- thorough peer review by experienced researchers in your field

- rapid publication on acceptance

- support for research data, including large and complex data types

- gold Open Access which fosters wider collaboration and increased citations

- maximum visibility for your research: over $100 \mathrm{M}$ website views per year 